\title{
MFM Study of the Domain Structure of Permalloy Microparticles under Mechanical Stress
}

\author{
Dmitry Biziyaev ${ }^{1}$, Anastas Bukharaev ${ }^{1,2}$, Niyaz Nurgazizov ${ }^{1,2 *}$ \\ ${ }^{1}$ Kazan E. K. Zavoisky Physical-Technical Institute, 420029 Kazan, Russia \\ ${ }^{2}$ Kazan Federal University, Institute of Physics, 420008 Kazan, Russia
}

\begin{abstract}
The domain structure of planar permalloy (Py) microparticles was studied under mechanical stress. An array of Py particles was formed by electron beam evaporation of Py on flat and preliminarily bent glass substrates. After evaporation the substrate was unbent and the Py particles were compressed along one axis. The change of the domain structure of stressed particles in comparison with that of unstressed particles was studied. It was shown that the change of the domain structure of Py particles depends on their compression ratio.
\end{abstract}

\section{Introduction}

Recently the possibility of using the Villari effect (the change of magnetic properties of a body under mechanical impact) for the magnetic reversal processes of micro and nanostructures is intensively studied [1-4]. This is due to the possibility of a significant reduction in the energy required for re-writing a single bit of information due to the simultaneous use of a magnetic field and mechanical stress.

Piezoelectric substrates, on the surface of which nanoparticles are formed, are usually used to create stress. Such substrates can change their geometric sizes under the action of an electric potential for compression or tension [4,5]. Another method of creating stress in particles is the elastic bending of the substrate. The substrates can be also bent before the particles are formed and to create stresses in the particles by straightening the substrate [2,6-9].

In addition, the change in the magnetic structure of micro- and nanoparticles upon their compression can be used to detect mechanical stress in solids. In this case, the lateral resolution of this technique will be comparable with the linear sizes of the nanoparticles under study. In this work the change in the magnetic properties of permalloy (Py) particles under mechanical compression was studied.

\section{Sample preparation}

The studies were carried out on samples representing an array of the planar Py (Ni79\%, Fe16\%, Mo4\%) particles (with the size of $25 \times 25 \times 0.03 \mu \mathrm{m}^{3}$ ) located on a glass substrate. Before forming the particles, the glass substrate was coated with a thin copper film $(\sim 5 \mathrm{~nm})$ for discharging the sample during measurements by the magnetic force microscope (MFM). The copper film and particles were fabricated by electron beam evaporation under ultrahigh vacuum conditions. The particles were deposited using a "Multiprobe P" device (Omicron). An array of identical particles was formed by sputtering through a metal grid placed on the surface of the substrate.

The optical image of obtained particles is shown in fig. 1a. The 3D-image of a single Py particle is shown in fig. 1b. Atomic force microscope (AFM) scanning of different parts of the sample made it possible to confirm the identity of all particles. The size of the used glass substrate was $12 \times 4 \mathrm{~mm}^{2}$, the thickness was $0.15 \mathrm{~mm}$. The region occupied with Py particles was in the center of the substrate and had the sizes of about $6 \times 4 \mathrm{~mm}$.

To create stressed particles, the substrate was elastically bent by fixing in the holder before conducting evaporation. After the substrate was straightened, the stresses were created in the particles. A sample holder with the constant curvature radius (of about $70 \mathrm{~mm}$ ) was used to create particles with the same degree of stress (fig. 2a). To create a sample with different compression ratios of particles along the long axis, an even holder was used, where a metal wire (with the diameter of $0.08 \mathrm{~mm}$ ) was placed under the substrate perpendicular to the axis (fig. 3a). When using a wire with the diameter of about $0.1 \mathrm{~mm}$ (or more), the substrate was broken. This meant that the non-uniform stresses produced in the glass during the creation of particles are close to the critical values.
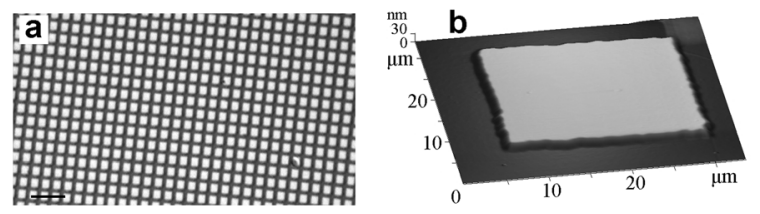

Fig. 1. Optical image of Py particles under study (a). The bar length is $0.1 \mathrm{~mm}$. AFM image of a single Py particle (b). 


\section{MFM studies of the domain structure of Py particles}

A Solver HV (NT MDT) device working in the MFM mode was used to study the domain structure of Py particles. The measurements were carried out in the onepass technique. In this case, the MFM tip did not affect the magnetization of Py particles. The $\mathrm{N} 18 / \mathrm{CoCr}$ (MikroScience) cantilevers were used. MFM scanning of the surface of the sample was carried out with a fixed step along the long axis of the sample (parallel to the compression direction of particles).

To detect the magnetization distribution in the particle, the MFM images (fig. 2b) were compared with results of computer simulation of its magnetic structure. These calculations were carried out by OOMMF [10] (fig. 2d). The following parameters were used: the exchange interaction constant of $1.3 \cdot 10^{-11} \mathrm{~J} / \mathrm{m}$, the unit cell size of $20 \mathrm{~nm}$ in the in-plane direction and $30 \mathrm{~nm}$ in the out-ofplane direction (equal to the thickness of the particle). The obtained magnetization distribution was used for simulation of the MFM image (fig. 2e) using the "Virtual MFM" software [11], which was compared with the experimental results. The coincidence of the model image with the experimental made it possible to make a conclusion about the correct calculation of the magnetization distribution.

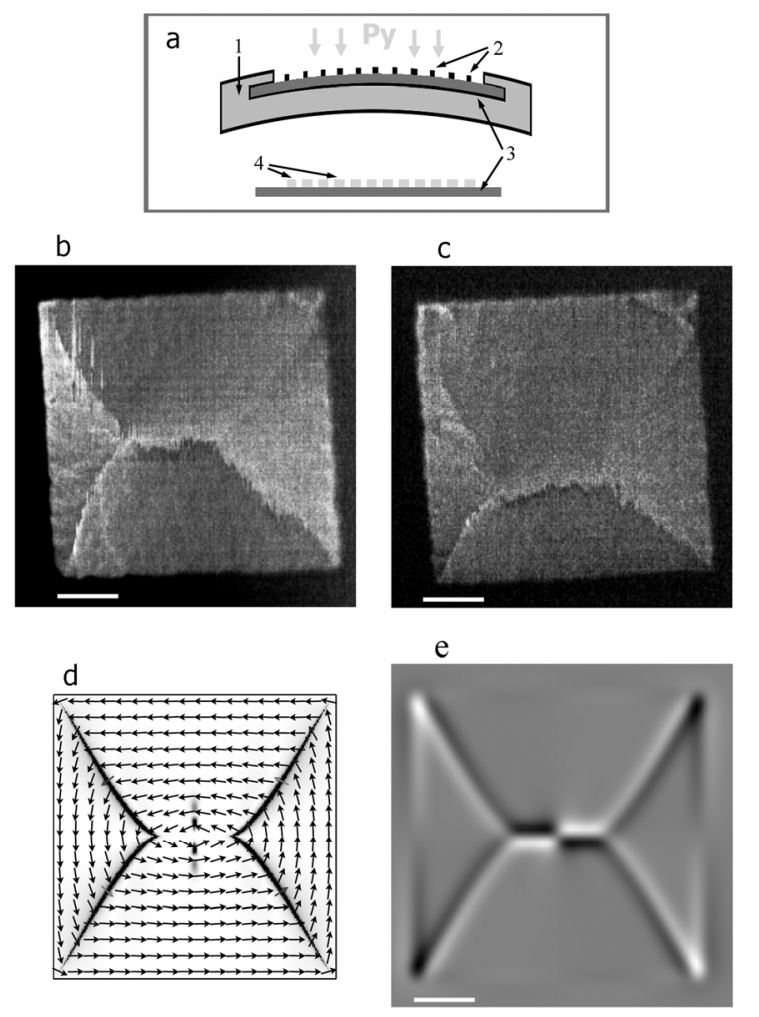

Fig. 2. Scheme for preparing a sample with the same compressed Py particles (a). Numbers denote: 1 - sample holder (with the constant curvature radius); 2 - metal grid; 3 substrate; 4 - Py particles. MFM images of Py particles obtained in different regions of the sample $(b, c)$. The particle magnetization simulated in OOMMF (d). The virtual MFM image calculated for such magnetization distribution (e). The bar length $-5 \mu \mathrm{m}$.
In order to achieve the coincidence of the model MFM images with the experimental ones (i.e., to find the magnetization distribution in the particle), two parameters were varied during the OOMMF calculation. The main varied parameter was the anisotropy coefficient $\left(\mathrm{k}_{\mathrm{eff}}\right)$. The second varied parameter was the saturation magnetization value $\left(8.6 \div 9.6 \cdot 10^{5} \mathrm{~A} / \mathrm{m}\right)$.

In our case, the anisotropy coefficient of the particle depends on the crystal anisotropy coefficient and the magnetoelastic anisotropy coefficient. The obtained Py particles had the polycrystalline structure therefore the anisotropy coefficient of the particle is determined by the magnetoelastic anisotropy coefficient. The anisotropy coefficient $\left(\mathrm{k}_{\text {eff }}\right)$ obtained by the calculations in the OOMMF program was used for the calculation of the effective anisotropy field $\left(\mathrm{H}_{\mathrm{a}}\right)$. The following formula $H_{a}=2 k_{\text {eff }} / M_{S}$, where $M_{s}$ is the saturation magnetization of the Py particle, was used. A similar approach was used in [3] to determine the magnetization distribution in $\mathrm{Ni}$ particles under mechanical stress.

MFM images obtained on uncompressed samples and in certain regions of a sample with differently compressed particles show that the uncompressed Py particles have the characteristic four-domain structure (fig. 3c). All domains have the same sizes and shape caused by the shape anisotropy. A small spread in the shape of the observed domains is due to the nonideal square shape of the prepared Py particles.

The change of domain structure was observed on samples with equally compressed Py particles (fig. $2 b, c)$. Two domains with the magnetization direction parallel to the compression direction increase in size (fig. 2d). Domains with the magnetization direction perpendicular to the compression direction decrease in size. This is due to the negative sign of the Py magnetostrictive constant.

A characteristic bridge between two large domains was observed on MFM images. Its length is proportional to the ratio between the areas of domains with the magnetization direction parallel to the compression axis and those with the magnetization direction perpendicular to the compression axis. This bridge length can be easily found from the MFM image of the particle and used for the comparison of the compression ratio of particles. The bridge length may be also used for the comparison of the experimental and model MFM images (fig. 2b,c and e).

For samples with identically compressed particles the same MFM images were observed over the whole sample. Obtained MFM images confirmed that in such samples all particles undergo the same stress. The effective anisotropy field of the Py particles due to uniform bending of the substrate during sputtering was $0.94 \mathrm{mT}$.

Three typical states of MFM images were observed on the samples with the different compression ratios of particles. In the regions near clumps (fig. 3a) the bridge on MFM images is perpendicular to the compression direction (fig. 3b). This indicates that the particles in this region are stretched. In the middle of the sample the bridge on the MFM image has the maximal length and is parallel to the compression direction (fig. 3e). It means that the particles in the center of the sample are maximally compressed. Since the Py particles are 
stretched at the edges of the sample and compressed in the center, they are unstressed between these regions. The effective anisotropy field of the Py particles varies to $3.12 \mathrm{mT}$.
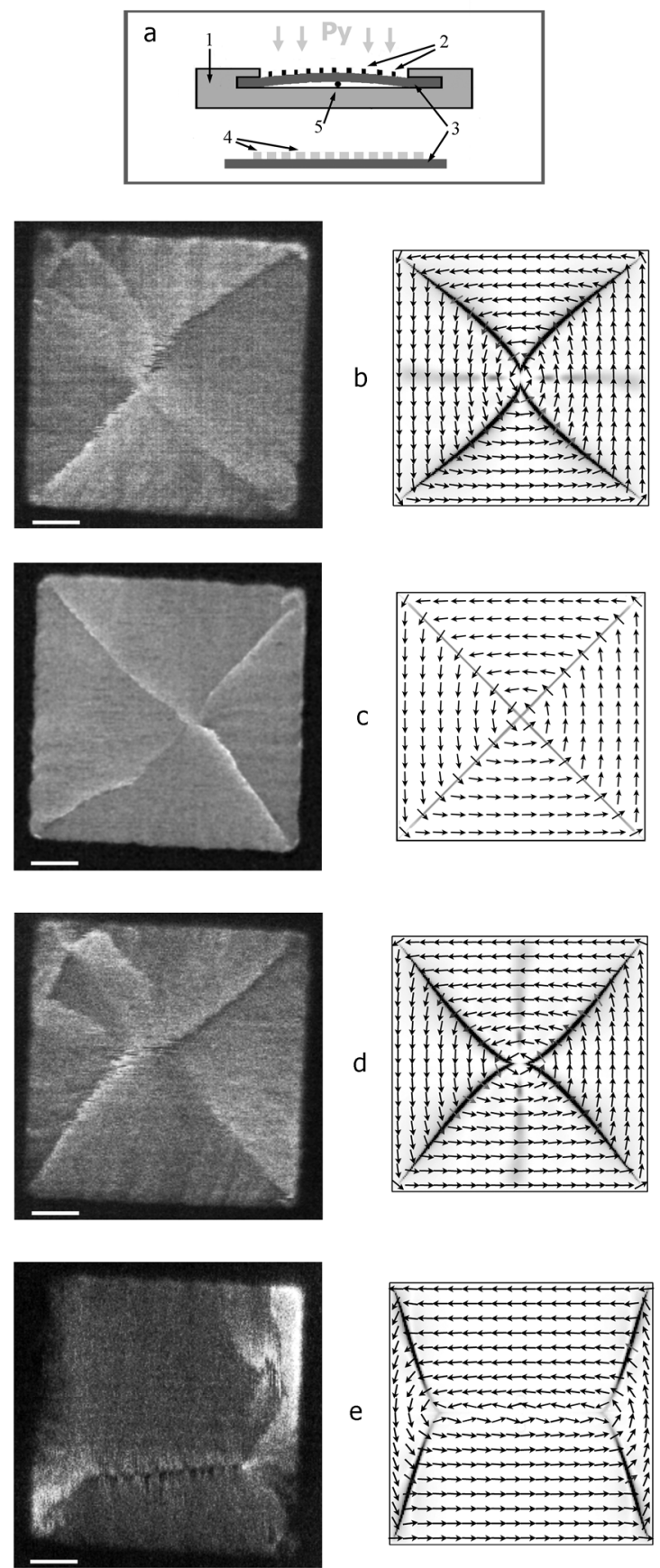

Fig. 3. Scheme of preparing of the sample with non-uniformly compressed Py particles (a). Numbers denote: 1 - sample holder (with the constant curvature radius); 2 - metal grid; 3 substrate; 4 - Py particles; 5 - Me wire for the creation of the compression gradient of particles. MFM images of Py particles with the corresponding magnetization simulated in OOMMF obtained at different distances from the sample edge: $0.3 \mathrm{~mm}$ (b); $0.9 \mathrm{~mm}$ - (c); $1.3 \mathrm{~mm}$ - (d); $2.9 \mathrm{~mm}$ - (e). The bar length is $5 \mu \mathrm{m}$.
According to the obtained MFM images, the "crosstie" structure was observed in highly compressed particles (fig. 3e). Such structure is formed at computer simulation in the case of the increase in the saturation magnetization value (up to $9.6 \cdot 10^{5} \mathrm{~A} / \mathrm{m}$ ). Ferromagnetic resonance studies carried out by us and other authors [5] showed that the saturation magnetization of Py particles may change under mechanical stress. It is possible that the "cross-tie" structure is formed in uniformly compressed Py particles resulting in the disappearance of the magnetic vortex in the centre of the particle (fig. 2c). At the same time the saturation magnetization almost does not affect the bridge length.

\section{Conclusions}

It was shown that uncompressed Py particles have the classical four-domain structure with domains of the same size. The uniaxial compression of particles results in the increase in the size of domains with the magnetization parallel to the compression direction (this is due to the negative Py magnetostrictive constant). The effective anisotropy field of $\mathrm{Py}$ particles with the same compression ratio was $0.94 \mathrm{mT}$.

It was established that the anisotropy effective field of the Py particles on non-uniformly curved samples changes to $3.12 \mathrm{mT}$. The particles are stretched at the edge of the sample and compressed in the center of the sample. The bridge length between domains was proportional to the compression ratio.

The work is supported by the Russian Foundation for Basic Research 17-08-00915.

\section{References}

1. Y. Liu, Q. Zhan, B. Wang, H. Li et al., IEEE Transactions on Magnetics, 51, 2501404 (2015)

2. G. Dai, Q. Zhan, H. Yang et al., J. Appl. Phys.114, 173913 (2013)

3. S. Finizio, M. Foerster, M. Buzzi et al., Phys. Rev. Applied, 1, 021001 (2014)

4. T. Wu, A. Bur, J.L. Hockel et al., IEEE Magnetics Letters, 2, 6000104 (2011).

5. A.I. Morozov, Physics of the Solid State 56, 865-872 (2014)

6. A. Bur, T. Wu, J. Hockel et al., J. Appl. Phys., 109, 123903 (2011)

7. Z. Hao, L. Yuan-Yuan, Y. Mei-Yin et al., Chinese Phys. B, 24, 07750 (2015).

8. A.P. Chuklanov, N.I. Nurgazizov, D.A. Bizyaev et al., Journal of Physics: C 714, 012006 (2016)

9. B.A. Belyaev, A.V. Izotov, Physics of the Solid State, 49, 1731-1739 (2007)

10. M.J. Donahue, D.G. Porter, OOMMF, (http://math.nist.gov/oommf/).

11. D.V. Ovchinnikov, A.A. Bukharaev, Technical Physics, 46, 1014-1019 (2001). 